\section{The employment of online questionnaires within user studies research in school libraries}

\author{
Andrew K. Shenton and \\ Andrew Johnson
}

\section{Authors}

Dr. Andrew K. Shenton is a former Lecturer in the Division of Information and Communication Studies at Northumbria University.

Andrew Johnson is Network Manager at the school where the study described in this article took place. Email:ashentonI@hotmail.com

\begin{abstract}
This paper considers the effectiveness of a research method based on online questionnaires and employed in a recent study of attitudes to books and school libraries among pupils in an English high school. The questionnaire was constructed in Microsoft SharePoint. In an e-mail, the youngsters were asked to complete the questionnaire and were taken to it via a hyperlink. The research strategy ensured that, in theory at least, a wide range of pupils was reached. It exploited their familiarity with electronic surveys and eliminated the problem of illegible handwriting often associated with paper questionnaires. Nevertheless, the response rate was poor and the quality of the data questionable despite several provisions for ensuring trustworthiness. The SharePoint system employed supported quantitative data analysis to a much greater extent than it did qualitative. The paper concludes by identifying other forms of electronic questionnaire that may be employed in schools that lack SharePoint software.
\end{abstract}

\section{Introduction}

For many years research has been undertaken in schools to investigate the reading habits and library inclinations of pupils. The methods employed have changed little during this time. Although one of the authors of this article has used an approach based on the recording of library inquiries in a transaction log (Shenton, in press), paper questionnaires and, to a lesser extent, interviews have formed the principal tools in the majority of published studies, even those that have been undertaken relatively recently in the current "electronic age". Paper questionnaires have featured, for example, in work described by Manson (1999) and Buckingham (2002), whilst a more qualitatively-oriented, interview-based approach has been applied by one of this article's authors, in a project with Dixon (Shenton and Dixon, 2002). In view of the substantial body of literature that testifies to the importance of electronic materials in relation to the information-seeking behaviour of young people, and the considerable popularity of computerbased activities among children and teenagers, it is perhaps surprising that so little research within schools seems to have utilised electronic data collection methods in investigations of pupils' views on books and libraries. Outside schools, there are notable precedents for their use. Indeed, one such study of young people's reading habits took place as long ago as October 1999 (Chance, 2000).

Obviously, if electronic techniques are to be used, much depends on schools having in place an appropriate and robust ICT infrastructure administered by a team of skilled technicians. For schools with flexible and powerful software which supports their intranet, one of the potential possibilities for the gathering of data electronically lies in the application of an online questionnaire. A recent study in a high school in the north-east of England has employed such a strategy and it is this work which forms the focus here. The piece does not aim to discuss the findings of the project. Rather, it explores the electronic data collection technique used. 


\section{Microsoft Sharepoint}

The organisation in which the study took place is a high school catering for between nine hundred and a thousand teenagers. It offers, via Microsoft SharePoint, an intranet available to staff, pupils and parents. Although the software itself is predominantly intended for document management in organisations, the school has adapted, for educational purposes, the functionality that it affords. In this context, the wide-ranging facilities provided by this software include the capability to

- allow teachers to upload school documents and learning resources for their easy access by those who need to consult them

- enable pupils to upload to their own user spaces material that they can consult and work with at home

- involve youngsters in producing their own self-assessments, subject targets and learning plans in response to prompts offered by a template, with the information produced stored centrally

- create hyperlinks to useful sites on the World Wide Web.

The most exciting opportunities for researchers, however, lie in the "survey" option that SharePoint affords. Here an investigator can create a questionnaire consisting of both openended and multiple choice questions and, ultimately, the document is presented as a web page mounted on the school's intranet.

\section{Strengths of the online questionnaire approach}

When considering the methods that could be used to collect data from the pupils with regard to their perspectives on books and the school library, SharePoint's "survey" facility appeared to provide the most satisfactory option. One of the foremost requirements of the approach was that the questionnaire should reach as many pupils as possible, including users of the school library and non-users alike. Manson (1999) reports how, in her research, feedback on the school library was collected via a paper questionnaire that was filled in by pupils during a form period. In the study addressed in this article, the researcher was reluctant to make such provision as it would have added to the administrative burden of teachers. In addition, if the questionnaire were to be completed during registration, a coercive element might be introduced, whereas it may be argued that data contributed voluntarily are more likely to be truthful. A further alternative - that of issuing questionnaires to pupils on their entry into the library - was rejected since youngsters who were non-users were just as important to the research as pupils who did exploit the facility. Even if library users had been exclusively targeted for the study, the distribution of questionnaires to each of them in this way would have proved impossible since both members of staff with responsibility for the library worked only part-time and there were key points during each week when the library was unmanned.

The construction of the questionnaire using SharePoint's "survey" facility proved straightforward even though the researcher had been hitherto unfamiliar with the function. The process was, no doubt, eased by the fact that he had already prepared a manual draft of the questionnaire and referred to this throughout the time that he spent generating an electronic counterpart. After creation of the computerbased questionnaire, a whole school distribution list was used to send each pupil an e-mail urging him or her to take part in the research. The message included a hyperlink to the address of the questionnaire. Clicking on this link took the user to the document itself, which could then be completed in minutes without any adult assistance. Figure 1 gives the reader some impression of how the questionnaire looked onscreen. The document could be filled in privately and at any time in the next month that was convenient to the recipient of the e-mail. Such privacy ensured that there was less opportunity for responses to be affected by peer group pressure than would have been the case with data collected in focus groups, for example, especially as a rule of no more than one person to each machine was applied in the computer room where it was envisaged that most of the questionnaires would be completed. Privacy was considered a particularly significant issue in view of the somewhat "dry" image of 
books and libraries among teenagers that has been highlighted by writers such as Dixon (1996) and Koch and Kendall (2003). Dixon
(1996) writes, "Young people... often fear peer disapproval if they express an interest in reading or other academic pursuits" (p. 54).

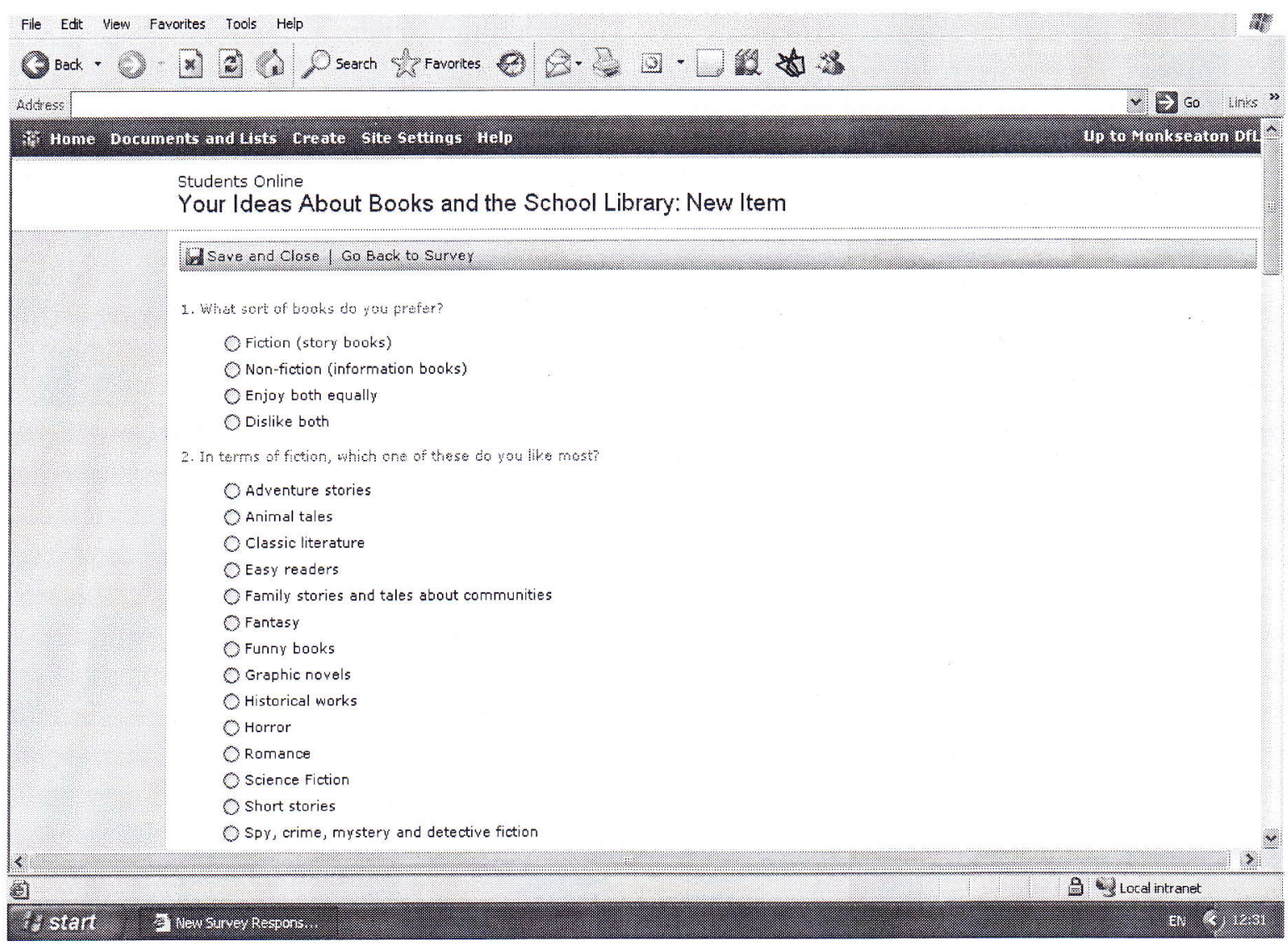

Figure 1: Extract from the questionnaire as it appeared on-screen.

The pupils' familiarity with electronic surveys increased the appeal in the eyes of the researcher of the approach that was taken. Coincidentally, as data for this study was being collected, pupils throughout the school were being asked to take part in a homework survey that was administered in a similar fashion, and all youngsters, including the Year Nines who had joined the school just a few months earlier, had already been required to use SharePoint's facilities to complete electronic statements of their attitudes to school and learning.

The computer-based questionnaire offered two further pragmatic benefits that were not provided by a paper equivalent. Firstly, it eliminated the problem of illegible handwriting, although, conversely, many errors that were due to poor keyboarding, as well as misspelling, emerged in the participants' responses.

Secondly, working in a wholly electronic environment afforded savings in terms of the cost of paper. These were considerable as, in this case, all the pupils in the school were targeted. The questionnaire was, however, relatively brief; had it been longer financial savings would have been even greater.

\section{Weaknesses of the method employed}

The ability of the online questionnaire method employed to reach a large number and wide range of pupils depended heavily, of course, on the frequency with which the youngsters read their e-mail. As e-mail provides a favourite method for many teachers in the school to communicate with their charges, all pupils are encouraged to check their school accounts regularly. Nevertheless, it was known that by no means everyone did this conscientiously. Unfavourable factors associated with pupil motivation may also have posed problems. 
When a pupil logs on to the school's computer system, it may be assumed that he or she does so for a certain purpose, typically to tackle academic work, to e-mail a friend, to play a game or to find information about a particular personal interest, maybe from a favourite website. Unless he or she has logged on specifically to check his or her school e-mails, the pupil may consider the receipt of a message asking that he or she completes a questionnaire for a school survey to be a distraction, and many of those reading it may have been tempted simply to delete the e-mail and resume their interest in their intended activity. With the message gone and the hyperlink to the questionnaire lost, it would be unlikely that the pupil would take part in the study at a later date. This weakness is symptomatic of a more general shortcoming that was identified in the method employed; whereas a paper questionnaire can be completed in a wide variety of situations and environments, its electronic counterpart has to be tackled online and if the document is not filled in fairly soon after the e-mail is read the task may well go undone.

With hindsight, a more effective strategy might have involved the creation of an additional link, on the pupils' personal home pages, to the online questionnaire. As this screen is the first that the user encounters when he or she opens Internet Explorer, it may be assumed that such a link is more likely to be seen than the e-mail sent by the researcher was to be read, although, of course, it, too, can still simply be ignored. Another option lies in using the Net Send command within Windows NT. This creates a message box which will pop up on the screen of a user to notify them of some information, in this case the survey. Sending one of these messages is quite easy and can be done by issuing a simple instruction in the command line. As Net Sends appear on the user's screen without being deliberately accessed, they are more intrusive than e-mails, however, and they can be employed only to alert users to a survey. Readers of the message are unable either to use any web address cited in it as a direct link or to copy and paste the address to the URL box of a browser. Had these opportunities been available, each would have provided a further route to the questionnaire itself. Another problem is that it is impossible to target a Net Send so that only pupils receive it. In this instance, a message could have been an irritation to teachers as the survey was not aimed at them.

Certainly, factors associated with the use of email in the survey's implementation may have been partly responsible for the survey's poor response rate of only around fourteen percent. This was despite the fact that as many as four weeks were allowed to pass between the questionnaire being posted on the intranet and the data being analysed. The response figure would have been even lower had a reminder not been sent to the pupils a fortnight after the original e-mail had alerted them to the survey. Whilst the low response rate may well have been due as much to lack of interest in the subject of the study as to shortcomings in the survey's implementation, with hindsight more might have been done to promote the research. All English teachers were asked if they could draw the attention of those in their classes to the survey but additional strategies such as publicising it via posters around the school and indeed in the library itself could have been adopted.

Concerns also emerged with regard to the quality of the data collected. In particular, it was impossible to determine how much thought and time participants had invested in providing their responses. Thus the trustworthiness of the data was open to question. Even where answers were offered with integrity, another danger arose. Most of the questions allowed a multiple choice system for responding and it was easy for pupils to mark an unintended answer, in much the same way as, in a manual questionnaire, mistakes can be made by carelessly ticking an undesired option.

Even though the online questionnaire was pilot tested, during the main phase of data collection it became apparent that the wording of two of the questions was insufficiently explicit and seemed to be giving rise to misunderstandings on the part of respondents. Knowing that the software would allow him to edit the questionnaire as the survey was still taking 
place and that, if changes were made, new participants would be working with the amended version, the researcher found himself facing a dilemma. If he left the questionnaire unaltered, undoubtedly more pupils would fall victim to the same misapprehensions as their predecessors but to modify the survey document at this stage would reduce the extent to which the new data and that already collected were comparable. Aware that neither course of action was ideal, the researcher took the first option.

\section{Provisions for trustworthiness}

Although the questionnaire did not itself require respondents to enter their personal details, whenever users logged on to the school's computer system they had to submit their usernames and passwords. For this survey, SharePoint recorded these details and stored them with the data that the participants contributed subsequently. The question of whether respondents were aware of this is an important one. If they knew that their replies could be traced back to named individuals, many pupils would, no doubt, have been encouraged to provide sensible answers, whereas those who believed that they were submitting the data anonymously might well have assumed a more frivolous attitude. Conversely, the counter-argument may be offered that frank responses are most likely to be given if anonymity is provided. In this instance, the decision was made to enable the researcher to trace data to named individuals but SharePoint also provides a facility for responses to be supplied anonymously. This is one of a range of ways in which the individual features of the survey can be customised to meet the researcher's needs. For example, by changing the settings the software also permits the investigator to allow only one questionnaire to be completed by a system user, or several. Since the former option was chosen, multiple submissions could not be made by the same individual unless he or she circumvented the restriction by logging on with the username and password of a pupil who had yet to respond to the survey. Such a scenario was unlikely, especially since all pupils knew that the school imposed strict penalties on anyone who logged on under the particulars of another user. An additional benefit was that the system recorded the date and time when each questionnaire was completed, thereby enabling the researcher to recognise situations where two or more pupils might have shared their ideas when working on their questionnaires.

\section{Data analysis}

The software proved better equipped to provide quantitative analysis than qualitative, and the effectiveness of even the former was largely restricted to data offered in reply to the multiple choice questions. This is perhaps a reflection of the fact that the software is not designed specifically to support major research projects. Nevertheless, one mode of analysis offered by SharePoint is the "graphical summary" option, which calculates frequencies and percentages for each of the answers to the questions and shows the results as simple bar charts. Once the first questionnaire had been submitted, these statistics could be produced at any time, thereby allowing the researcher to gain some feel for emergent patterns and trends as the questionnaires were being received. It was also possible for him to delete questionnaires completed during the pilot study and for the frequency statistics to be generated purely from the data contributed during the main phase of data collection. In the same way, any questionnaires that provided data deemed untrustworthy could be eliminated and, again, frequency calculations could be made on the basis of the remainder.

\section{Whilst the "graphical summary" function} enabled overall patterns within the data offered in response to multiple choice questions to be identified speedily, problems arose when the participants were allowed to submit their own text. In response to a question about favourite writers, Tolkien was variously entered as "JRR Tolkien", "J.R.R.Tolkien" and "J R R Tolkien", and the system treated each as a separate entity. A similar issue emerged when the name, Jacqueline Wilson, was misspelled, thus producing several variants. In these situations, frequencies had to be determined by manually adding together the numbers of instances of all 
such variants. Another analysis option available to the researcher was that of exporting the data to a Microsoft Excel spreadsheet. This option was not pursued yet it would, nonetheless, have allowed the researcher to employ the "countif" function to search for "*Tolkien*" and generate a count for all instances of the surname, regardless of the different ways in which the writer's initials had been represented. Any spreadsheet to which the questionnaire data is downloaded can also be imported into a Microsoft Access database. Clearly, if searching of this database is to be effective, however, the entries have to be carefully checked for correct spelling. At present, the software provides no facilities for the categorising and coding of qualitative data. Any data of this kind that is elicited must, therefore, be analysed either manually or via dedicated software. Still, it is possible for the text entered by individual respondents to be copied and pasted from the questionnaires into a Microsoft Word file, thereby enabling the researcher to quote to the reader of the ultimate report the exact language of a participant without any danger of errors in transcription.

\section{Alternative approaches}

By no means every school, especially at primary level, is equipped with the facilities necessary for constructing the kind of electronic questionnaire that has been described here. One alternative lies in creating a more traditional type of questionnaire using Word, e-mailing the document to pupils within the targeted group via a predefined distribution list and asking each recipient to complete the questionnaire before emailing it back to the researcher. Although clearly much of the responsibility for analysing the data in these circumstances will fall back on the researcher personally as there is no opportunity for automated frequency counts to be generated, and the researcher will be faced with the unenviable task of extracting data from a collection of individual documents, the method at least offers some of the advantages associated with the technique described in this article. In particular, youngsters are again given the opportunity to work in the electronic environment that has become so familiar to them, a large number of pupils will be reached and savings on the cost of paper are likely to be considerable.

A further alternative lies in creating the questionnaire via Excel. This may be as a form that incorporates familiar features of graphical user interfaces, such as drop-down boxes. After participants have entered their data and submitted their documents, each user's questionnaire can be treated as a separate workbook. Ultimately, the researcher may construct a final workbook from the collection of workbooks so that data from the originals can be merged and aggregated. This method allows the drudgery associated with making frequency counts to be shifted, again, to the computer but inevitably the work demands considerable expertise in constructing the questionnaire in Excel. Whereas many school librarians would, no doubt, feel comfortable creating a questionnaire in Word, they might be rather less at ease when setting up an equivalent in Excel.

\section{Conclusions}

Even in today's era in which computers impinge on so many areas of young people's lives, including their information-seeking, the use of electronic questionnaires in schools to collect data on the attitudes of pupils to books and libraries remains infrequent. For schools with well developed ICT infrastructures and appropriate levels of technical support, the gathering of data in this way can, however, offer appealing possibilities. Electronic questionnaires, which may be created in Word or Excel, as well as via document management software such as SharePoint, may exploit young people's enthusiasm for and familiarity with computers, although this in itself does not guarantee a high response rate, of course. A key consideration would appear to lie in the nature of the strategy that is used to bring the questionnaire to the pupils' attention. As with paper questionnaires, doubts may emerge with regard to the quality of the data elicited, since, in both contexts, careless mistakes can easily be made when completing the questionnaire and respondents may be tempted to rush their answers simply to finish the task speedily. 
Furthermore, if early responses reveal problems in the design of the questionnaire, the researcher is faced with the dilemma of whether to make changes to it whilst the study is in progress. Problems may arise, too, from the limitations of the software used to create the questionnaire and to mount it on the school's intranet. The software employed in the project described in this paper provided no facilities for analysing qualitative data and even its facilities for analysing quantitative data were rudimentary.

\section{References}

Buckingham, R. (2002) Highlighting the importance of reading and literacy in secondary school LRCs: a case study, School Librarian 50(1), 10-13.

Chance, R. (2000) SmartGirl.com reading survey: what are the messages for librarians? Journal of Youth Services in Libraries I3(3), 20-23.

Dixon, J. (1996) Are we childproofing our public libraries? Identifying the barriers that limit library use by children, Public Libraries 35(I), 50-56.

Koch, L. and Kendall, M. (2003) Older teenagers' attitudes to a Further Education college library's fiction collection, New Review of Children's Literature and Librarianship 9, 21-40.

Manson, S. (1999) The user's perception of the school library, School Librarian 47(2), 68-69.

Shenton, A. K. [in press] The role of 'reactive classification' in relation to fiction collections in school libraries, New Review of Children's Literature and Librarianship.

Shenton, A. K. and Dixon, P. (2002) Youngsters' use of and attitudes to their school libraries: results of a qualitative research project, School Librarian 50(4), 176-78. 